\title{
Comparative Performance Analysis of a Brownian Carnot Cycle from the Perspective of a Stochastic Model against the Linear Irreversible Thermodynamics Theory ${ }^{\dagger}$
}

\author{
Ricardo T Paez Páez-Hernández ${ }^{1}$, Juan Carlos Chimal-Eguía ${ }^{2}$ and Juan Carlos Pacheco-Paez 1,3 \\ 1 Universidad Autonoma Metropolitana u Azcapotzalco Av. San Pablo No. 180 Col. Reynosa Tamaulipas, \\ Ciudad de México, Mexico \\ 2 Centro de Investigación en Computación del IPN, Av. Juan de Dios Batiz s/n esq Miguel Othón de \\ Mendizabal, col. San Pedro Zacatenco, Ciudad de México, Mexico \\ 3 Department of Applied Mathematics and Computing, Faculty of Higher Studies, Division of Mathematics in \\ Engineering, UNAM, Mexico \\ † Presented at the Entropy 2021: The Scientific Tool of the 21st Century, 5-7 May 2021; Available online: \\ https://sciforum.net/conference/Entropy2021/.
}

Published: 5 May 2021

In this work we present a Brownian Carnot Cycle, which has already been studied by Schmield et al. (2007) as well as Izumida and Okuda (2010); but now considering two different woring regimes, namely the Maximum Ecological Function (MEF) and the Maximum Efficient Power (MEP). Fort he MEF and MEP working regimes, the thermodynamic properties of the cycle are obtained, in particular, it showed that the máximum efficiency now depends on two parameters $\alpha$ and $\beta$, instead of only one parameter obtained previously by Schmield et al. In máximum power regime. It is worthwhile to notice that for characteristic values of $\alpha$ and $\beta$ the original results obtained by Schmield are recovered.

From the previous observations, the authors consider that the results obtained represent a more general case that includes other working regimes. It is important remark that one of the most astonishing results obtained, is that those thermal engine models show some universality regarding the behavior of the efficiency when it works at the maximum power regime, although the analyzed models were different in nature and scale.

In this work we present a Brownian Carnot Cycle, which has already been studied by Schmield et al. (2007) as well as Izumida and Okuda (2010); but now considering two different woring regimes, namely the Maximum Ecological Function (MEF) and the Maximum Efficient Power (MEP). For the MEF and MEP working regimes, the thermodynamic properties of the cycle are obtained, in particular, it showed that the máximum efficiency now depends on two parameters $\alpha$ and $\beta$, instead of only one parameter obtained previously by Schmield et al. In máximum power regime. It is worthwhile to notice that for characteristic values of $\alpha$ and $\beta$ the original results obtained by Schmield are recovered.

From the previous observations, the authors consider that the results obtained represent a more general case that includes other working regimes. is important remark that one of the most astonishing results obtained, is that those thermal engine models show some universality regarding the behavior of the efficiency when it works at the maximum power regime [1], although the analyzed models were different in nature and scale.

\section{Reference}

1. Norma Sanchez-Salas, L López-Palacios, S Velasco, A Calvo Hernández, Optimization criteria, bounds, and efficiencies of heat engines. Phys. Rev. E 2010, 82, 051101. 
(C) 2021 by the authors. Licensee MDPI, Basel, Switzerland. This article is an open access article distributed under the terms and conditions of the Creative Commons Attribution (CC BY) license (http://creativecommons.org/licenses/by/4.0/). 